\title{
Statistical Tests for Detection of Frequency-Modulated Signals
}

\author{
Kian Blanchette ${ }^{1}$, Wesley Burr ${ }^{2}$, Glen Takahara ${ }^{1}$ \\ ${ }^{1}$ Department of Mathematics \& Statistics, Queen's University \\ 99 University Avenue, Kingston, Canada \\ 18kmlb@queensu.ca; takahara@queensu.ca \\ ${ }^{2}$ Department of Mathematics, Trent University \\ 1600 West Bank Drive, Peterborough, Canada \\ wesleyburr@trentu.ca
}

\section{Extended Abstract}

The detection of periodic signals in time-series data is a classic problem in statistics, and is critical to scientific development in many fields, from oceanography to helioseismology. The multitaper-based Harmonic F-test Statistic [1] is an effective, robust tool for detection of such signals, although its performance degrades in the presence of frequency modulation. Helioseismology in particular has data which is particularly prone to such frequency modulation, with many signals being evident in such data with rotation or orbital frequency modulation [2], [3]. The development of robust tests for frequency-modulated signals is thus of high interest to many applied science fields, for use on high-value scientific data sets.

In [4], Thomson described a family of projection filters that pass polynomials. These filters linearly combine the discrete prolate spheroidal sequences [5], with a unique (up to the length of the data, N, and the multitaper bandwidth, W) set of associated polynomials. Thomson then shows that these projection filters [6], when fit to low-order polynomials to a time-series' estimated instantaneous frequency in the neighbourhood of arbitrary carrier frequencies, give a reasonable approximation to any existing frequency modulation, allowing for demodulation of the series around the carrier. Along with the polynomial fitting procedure, Thomson proposed a test statistic for selecting the best order of polynomial for the fit in [6], and (personal communication) also derived an alternative statistic. These two test statistics are approximately distributed as $\mathrm{F}(\mathrm{P}, \mathrm{K}-\mathrm{P})$ random variables, with $\mathrm{K}$ the number of instantaneous frequency eigencoefficients (effectively, the frequency domain sample size) and $\mathrm{P}$ is one more than the degree of the polynomial.

In this work we propose an additional test statistic, distributed as an $\mathrm{F}(1, \mathrm{~K}-\mathrm{P})$ random variable and consider using these test statistics as a method for detecting frequency modulated signals and estimating their carrier frequencies by carrying out the test statistic procedure across the entire principal frequency domain at once in a similar fashion to the Harmonic F-test statistic.

We use simulation studies to compare the performance of the three polynomial test statistics, compared against the Harmonic F-test statistic and each other. In the simulations, we consider 10,000 iterations of the classic sinusoid plus noise case, each of length $\mathrm{N}=2048$, center sinusoidal frequency $\mathrm{f}=0.2$, and quadratic instantaneous frequency embedded in additive Gaussian white noise. The Thomson-proposed test statistics correctly obtained a maximum greater than the 1-1/N quantile of the $\mathrm{F}(3, \mathrm{~K}-3)$ distribution in $(\mathrm{f}-\mathrm{W}, \mathrm{f}+\mathrm{W})$ about $48 \%$ of the time. They also incorrectly achieved significant maximums in (f-W, $\mathrm{f}+\mathrm{W})$ at degrees 1,3 and 4 in roughly $5 \%, 35 \%$ and $30 \%$ of the simulations, respectively. The classic Harmonic F-test statistic achieved a maximum in-band just 9 times out of the 10,000, essentially negligible. The degree 2 $\mathrm{F}(1, \mathrm{~K}-\mathrm{P})$ test statistic that we proposed achieved an in-band maximum at the above significance level in roughly $75 \%$ of the simulations, while the degree 1,3 and $4 \mathrm{~F}(1, \mathrm{~K}-\mathrm{P})$ statistics incorrectly achieved in-band maximums 8,15 and 437 times, respectively. Further examination of this and other aspects of performance, such as robustness to nonstationarity and non-Gaussianity will be discussed. 


\section{References}

[1] D. Thomson, "Spectrum estimation and harmonic analysis," Proceedings of the IEEE, vol. 70, pp. 1055-1096, 1982.

[2] D. Thomson, "Spectra of MDI magnetograms," in Proc. of the SOHO 14 / GONG 2004 Workshop, 'Helio-and Asteroseismology: Towards a Golden Future', (Noordwijk), pp. 91-100, European Space Agency, SP-559, 2004.

[3] R. A. García, A. Jiménez, S. Mathur, J. Ballot, A. Eff-Darwich, S. J. Jiménez-Reyes, P. L. Palle, J. Provost, and S. Turck-Chièze, "Update on g-mode research," Astron. Nachr., vol. 329, pp. 476-484, 2008.

[4] D. Thomson, "Inverse-constrained projection filters," Proceedings SPIE, vol. 4478, pp. 172-183, 2001.

[5] D. Slepian, "Prolate Spheroidal Wave Functions, Fourier Analysis, and Uncertainty V: the Discrete Case," Bell System Technical Journal, vol. 57, pp. 1371-1429, 1978.

[6] D. Thomson, "Polynomial phase demodulation in multitaper analysis," in SSP '09 IEEE/SP 15th Workshop on Statistical Processing, (Cardiff), pp. 401-404, IEEE Press, 2009. 